\title{
Bericht aus dem Arbeitskreis Gerontopsychiatrie
}

\section{Gerontopsychiatrie in Zeiten von COVID 19}

Vom 7. bis 8. November 2019 tagte die Arbeitsgruppe Gerontopsychiatrie der Bundesdirektorenkonferenz im AlexianerKrankenhaus Hedwigshöhe in Berlin bei Frau Professor Dr. V. Holthoff-Detto.

Neben der Vorstellung verschiedener Projekte in der Gerontopsychiatrie wurde eingehend die Personalsituation in den gerontopsychiatrischen Einrichtungen diskutiert. Es zeigen sich deutliche regionale Unterschiede in der personellen Besetzung. In vielen Kliniken mangelt es nicht nur an Weiterbildungsassistenten, sondern es besteht auch ein deutlicher Fachärztemangel. Dieses führt in einigen Regionen zu Schwierigkeiten, eine gerontopsychiatrische Versorgung in stationären Pflegeeinrichtungen über die Institutsambulanzen sicherzustellen. Unbesetzte Stellen, die Mehrbelastung für die Kolleginnen und Kollegen und eine hohe Fluktuation in der Ärzteschaft erschweren Konzeptarbeit und Teambildung. Gerade zukunftsorientierte Behandlungskonzepte in der Gerontopsychiatrie, wie die stationsäquivalente Behandlung (StäB), können nur von erfahrenen Fachkräften mit ausreichend langer Berufserfahrung geleistet werden.

Weitere Themen der Herbsttagung waren die nationale Demenzstrategie, eine bundesweite Erfassung der Gerontopsychiatrischen Zentren und Gedächtnissprechstunden, die Auswirkungen des neuen MDKGesetzes für die Gerontopsychiatrie sowie die PPP-RL.

Die für Mai 2020 geplante Frühjahrstagung in Stralsund musste aufgrund der SARS-CoV-2-Pandemie abgesagt werden. Die Auswirkungen dieser Pandemie für die Behandlung und Pflege psychisch kranker alter Menschen sind gegenwärtig noch nicht abzuschätzen. Viele Kliniken mussten sich sehr schnell auf Isolierungs- und Quarantänemaßnahmen einstellen, für die aber in der Regel keine baulichen Vorkehrungen bestehen. Für Menschen mit Demenz, die infolge ihrer Desorientierung und oft in Verbindung mit einem starken Bewegungsdrang („Hinlauftendenz“) besondere personelle, räumliche und strukturelle Versorgungsstrukturen benötigen, ist eine Isolation einfach nicht so umsetzbar, wie bei psychisch gesunden Menschen. Auch die Gesundheitsbehörden haben derzeit keine wirklich effektiven Lösungsvorschläge, wie Quarantäne und Isolation auf Stationen und in Wohnbereichen für diese Menschen umgesetzt werden könnte. In der Regel bleibt es bei pragmatischen Lösungen und Kohortenisolierungen. Auch lassen sich HygieneInstruktionen (z.B. Tragen von MundNase-Schutz, Händedesinfektion, Abstandsregeln) hier nicht realisieren. Die zusätzlich verhängten Besuchssperren, die zum Schutz vor Ansteckungen mit SARS-CoV-2 verfügt wurden, stellen für psychisch erkrankte ältere Menschen eine schwere Belastung dar. Die Einsamkeit dieser Menschen wird durch die Anordnung von Isolationsmaßnahmen noch verschärft, die Folgen für die seelische Gesundheit sind kaum vorhersehbar.

Als besondere Herausforderung entpuppt sich in diesem Zusammenhang nun die Entlassung von älteren Menschen nach Abschluss gerontopsychiatrischer Behandlung in stationäre Pflegeeinrichtungen und in die ambulante Versorgung. Durch die Schließung von Tagespflegen ist eine Säule der Versorgung komplett weggebrochen. Die - als Beispiel für diverse Regelungen in den Bundesländern - vom Ministerium für Arbeit, Gesundheit und Soziales des Landes Nordrhein-Westfalen (MAGS NRW) verfügte 14-tägige Quarantäne nach Neuaufnahme eines Heimbe- wohners, mag aus seuchenmedizinischen Gründen nachvollziehbar sein, widerspricht aber einer sinnvollen Integration in ein neues Wohnumfeld: Gerade nach der Aufnahme in eine stationäre Pflegeeinrichtung spielt die Begegnung mit Mitbewohnerinnen und Mitbewohnern sowie die Einbindung in gemeinschaftliche Aktivitäten eine zentrale Rolle. Gerontopsychiatrische Kliniken wie auch stationäre Pflegeeinrichtungen werden in der SARSCoV-2-Pandemie vor große Herausforderungen gestellt: Die Umsetzung von $\mathrm{Hy}-$ gienemaßnahmen bei Patienten ohne jegliche Krankheitseinsicht, bei Abwehr von Pflegemaßnahmen und Umherlauftendenz sind oft schlicht nicht realisierbar und bedürfen einer Umsetzung mit Augenmaß. Die Lösung kann nicht per Erlass erzielt werden. Umso deutlicher wird der Stellenwert von gerontopsychiatrisch qualifiziertem Fachpersonal in dieser Krisensituation. Wünschenswert wäre gewesen, dass Pflegeheime und gerontopsychiatrische Fachabteilungen frühzeitig mit Schutzausrüstung ausgestattet worden wären. Wegen vermeintlicher Versäumnisse in einzelnen Einrichtungen trat stattdessen die Staatsanwaltschaft in Erscheinung. Zwar hört man derzeit oft Worte der Anerkennung für die Leistungen von Menschen in den Gesundheits- und Pflegeberufen. Aber wird es zu nachhaltigen strukturellen Verbesserungen führen? Hoffen wir, dass nach Abklingen der Pandemie die Arbeit von Menschen, die unter diesen schwierigen Umständen in der gerontopsychiatrischen Versorgung arbeiten, neu bewertet wird.

\section{Korrespondenzadresse}

Prof. Dr. med. Tillmann Supprian

Abteilung Gerontopsychiatrie, LVR-Klinikum Düsseldorf, Kliniken der Heinrich-Heine-Universität Düsseldorf

Bergische Landstraße 2

40629 Düsseldorf

E-Mail: tillmann.supprian@lvr.de 